\title{
Expression of Emotional States during Locomotion based on Canonical Parameters
}

\author{
Martin Inderbitzin, Aleksander Väljamäe, Jose Maria Blanco Calvo \\ Paul F.M.J. Verschure and Ulysses Bernardet
}

\begin{abstract}
Humans have the ability to use a complex code of non-verbal behavior to communicate their internal states to others. Conversely, the understanding of intentions and emotions of others is a fundamental aspect of human social interaction. In the study presented here we investigate how people perceive the expression of emotional states based on the observation of different styles of locomotion. Our goal is to find a small set of canonical parameters that allow to control a wide range of emotional expressions. We generated different classes of walking behavior by varying the head/torso inclination, the walking speed, and the viewing angle of an animation of a virtual character. 18 subjects rated the observed walking person using the two-dimensional circumplex model of arousal and valence. The results show that, independent of the viewing angle, participants perceived distinct states of arousal and valence. Moreover, we could show that parametrized body posture codes emotional states, irrespective of the contextual influence or facial expressions. These findings suggest that human locomotion transmits basic emotional cues that can be directly related to canonical parameters of different dimensions of the expressive behavior. These findings are important as they allow us to build virtual characters whose emotional expression is recognizable at large distance and during extended periods of time.
\end{abstract}

\section{INTRODUCTION}

The ability to understand others emotional intention, communicated solely by non-verbal cues, has a clear evolutionary advantage which is reflected in the social capability of an individual [1], [2]. Emotional states are the result of a complex multidimensional mechanism that integrates the perception of external stimuli with subjective internal needs [3], [4], [5]. The result is an active or passive response often accompanied by expressive components, such as facial expressions, vocalizations, body movements or physiological reactions. It has been shown that expressive behavior, especially facial expressions, can directly be related to internal emotional states, that are coded by universal, culturally unaffected schemata [6], [7], [8], [9]. Recent studies have shown similar results for verbal and non-verbal emotional vocalizations [10],

M. Inderbitzin, A. Valjamae, J.M. Blanco Calvo, P.F.M.J. Verschure and U. Bernardet are with the Laboratory of Synthetic Perceptive, Emotive, and Cognitive Systems - SPECS, Universitat Pompeu Fabra, Roc Boronat 138, E-08018 Barcelona, Spain martin.inderbitzin@upf.edu

P.F.M.J. Verschure is with the Catalan Institute of Research and Advanced Studies - ICRE paul. verschuredupf.edu
[11]. Yet, emotional behavior does not only express emotional states; different studies have questioned this view, claiming that non-verbal emotional expressions are mainly a social construct, rather than expressing purely internal emotional states [12], [13]. These studies show that humans use non-verbal emotional body language mainly to establish social relations and interactions. Hence, emotional expressive behavior can have two main functions: Firstly, the communication of internal emotional states, and secondly, to foster social relationships and hierarchical structures.

One major challenge for the understanding of the meaning of expressive behavior is to find a schematic classification. This is not a trivial task, that motivates researchers from sociology, behavioral psychology, theater, and dance studies since decades. While a great deal of attention was focused on the understanding and classification of emotional facial expressions [14], [6], [7], [8], [9], relationally little systematic research has been carried out in the field of emotional body language. One approach to describe body movements is the Laban Movement Analysis (LMA), that divides expressive biological motion into four different dimensions: Body, Effort, Shape and Space [15], [16]. Using its own symbolic notation, this analysis method is capable of specifically describing body movements. The LMA is a powerful tool that can be used for the production and especially the reproduction of human behavior in acting and dance. Despite these capabilities, the LMA lacks a clear linkage between behavior and emotions. Another theoretical concept is Bridwhistell's theory of kinesics that understands the language of the human body as a "structured dynamic process of communication“" [17], [18]. Unfortunately, the results of his extensive studies are not systematical ordered and thereby difficult to quantify [19]. A simpler classification system was proposed by Mehrabian, focusing on the orientation of the head in relation to the body and the angles of bodies interacting with each other [20].

What all classification systems have in common is a difficulty to find a direct relationship between the affective state and concrete corporal configuration and body movement. In contrast to facial expressions, where we can observe coherent relationships between basic emotions and expressive behavior [6], the inter- 
pretation of expressive body behavior is more sensitive to contextual and social influences [21]. Nevertheless, there is some empirical evidence that the movement and the form of the human body communicates emotions [22], [23], [24], also at a distance where facial expression is not detectable [25]. A promising approach is to analyze the emotion attributing of predefined body postures or movements, and correlate them with the parameters defining the body configuration. Studies following this idea differ methodological by either exposing viewers to real actors playing [23], video scene of actors playing [2], computer animations of virtual humans [26], [27], point-light animations that conceptualize human body movements [28], [29], [30], [31], [22], or drawn figures [32]. The results of these and similar studies show that affective states can be identified by observing static postures [33], [26], [34], [35] or moving behavior [36], [37], [23], [2].

The exact contribution of form and movement for the perception of emotional states is the topic of an extended discussion in the field. A recent study by Roehter et al. states that the understanding of affective body language is an integrative process of the perception of both dimensions, form and movement [38]. They identify the limb flexion velocity as an important feature for the perception of fear and anger, while the upper body posture, especially the head inclination communicates a sadness. These results are in line with a study from Thurman et al that investigates the perception of different critical features for biological motion [39]. Exaggerated body movement facilitates the recognition of affective states, especially the intensity of them [40]. On the other side the contribution of the form dimension for the identification of emotional states was made visible by a study using inverted and reversely played sequences of a moving person [41]. The result of these studies can be interpreted that the form plays a crucial role in affect identification, while kinetics help to solve conflicts and the identification of the intensity of the emotion. A finding that is in line with perceptual studies investigating the neurobiological mechanism of motion perception [42].

The emotional classifications used to describe affective behavior differ in complexity. The basic emotion approach is claiming that there exist a finite set of distinguishable emotions that can be attributed to expressive behavior [43], [44], [45], [46]. The numbers of proposed basic emotions vary between $4-10$, including happiness, fear, sadness, surprise, disgust, anger, joy, interest, elation, subjection, tender-emotion, wonder, contempt, guilt, and shame. The dimensional approach to emotions describes affective states using a two-dimensional classification system known as the circumplex model [47], [48]. This theory provides a circular classification space of basic emotions using valence and arousal to describe the quality and intensity of different emotional states. Both systems are used to describe expressive body movements [26], [2].

The purpose of the study presented here is to find a set of canonical parameters that control the expression of emotions in locomotion animations that are not dependent on facial expressions. The motivation is that we want to construct believable interactive characters for immersive virtual environments and mixed reality spaces, we need to investigate the emotional meaning of active behavior that is also perceivable at distance; virtual humans that interact with physical humans in a closed loop scenario only become realistic if we understand the emotional meaning of animated behavior, not only looking at fixed postures. Here we present the results of how people perceive the emotional state of a person walking. Based on the results of previous studies [26], [2], [32], we constructed different animations of expressive locomotion by varying three parameters of the movement: The head/torso inclination, including the erection of the shoulder, the speed of the movement, and the viewing angle.

\section{METHODS}

\section{A. Participants}

We selected 18 participants from the University Pompeu Fabra for our study. All the participants were either master students, $\mathrm{PhD}$ students or professionals working in academic and were permanent living in Spain. The mean age of the participants was 28.4 years $(\mathrm{SD}=4.3 ; \mathrm{M}=70 \% ; \mathrm{W}=30 \%)$.

\section{B. Materials}

The animations were modeled using Autodesk 3ds Max [49] and transferred to the Torque Game Engine [50]. As stimuli we exported from Torque 10 sequences of a length of 10 seconds each. For the stimuli exposure, and the rating of the sequences we used a 15 inch IBM Think Pad Laptop running the Eprime1 experiment exposure software [51]. The selfassessment manikin rating scale [52] was used for the evaluation of the sequences.

\section{Stimuli Design}

We constructed 12 different animations of a person walking by varying the parameter of the head-torso inclination, the speed of the movement, and the viewing angel (Figure 1). We defined the head-torso inclination of the neutral body posture as inclination angle zero, and used this as a reference for the other animations. The deviation of the head-torso varied between -55 and +15 degrees. The convention applied was that minus inclination indicates a ventral direction, positive declination a dorsal one. Half of the animations were showing the walking body in profile view (90 degree 


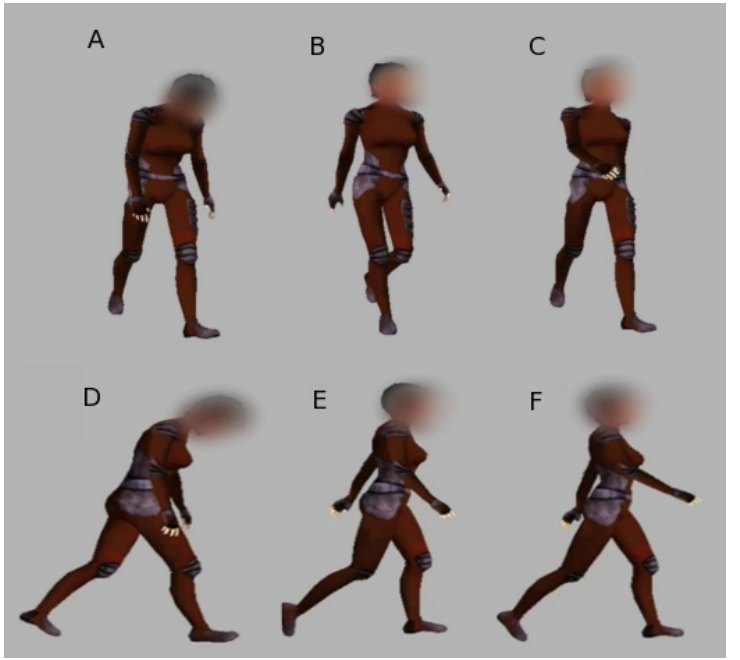

Fig. 1. Still images of stimuli in frontal view (A-C), and side view (D-F). Head/Torso inclination varied between 55 degree down (A, D), zero degrees (B, E), and 15 degrees up $(C, F)$.

viewing angle), the other half in 45 degree rotated frontal view (Table I). The animated avatar was a women wearing a dark, red-blackish suit, and dark shoes. To avoid any contextual influence we used a neutral gray color as background [53], [21]. The face of the character was blurred to avoid any influence of the facial expression [54], [55].

TABLE I

SPECIFICATION OF THE STIMULI PARAMETERS

\begin{tabular}{|c|c|c|}
\hline Viewing Angel & Inclination [Degree] & Speed [steps/sec] \\
\hline 45 & Neutral [0] & Medium [0.75] \\
90 & Neutral [0] & Medium [0.75] \\
45 & Up [+ 15] & Medium [0.75] \\
90 & Up [+ 15] & Medium [0.75] \\
45 & Down [- 55] & Medium [0.75] \\
90 & Down [- 55] & Medium [0.75] \\
45 & Neutral [0] & Slow [0.5] \\
90 & Neutral [0] & Slow [0.5] \\
45 & Neutral [0] & Fast [1.4] \\
90 & Neutral [0] & Fast [1.4] \\
\hline
\end{tabular}

\section{Procedure}

Participants were sitting alone at a table in front of a computer laptop used for the stimuli presentation, and asked to rate the valence and arousal state of a walking person. Each sequence was played for 10 seconds, followed by a black screen. After 2 seconds the valence and arousal rating scale appeared until a rating was given. The pause before the next stimulus sequence was played was 4 second. The order of the sequences was randomized. After the experiment, participants were asked by the experimenter if they had any problems to follow the experiment. Participants were not informed about the specific objective of the study.

\section{RESULTS}

The data was analyzed using the SPSS software package. The valence and arousal ratings were submitted to two multivariate analysis of variance (MANOVAs) where Wilks Lambda was used as the multivariate criterion. The first MANOVA factors were 2 (viewing angle) x 3 (head inclination), and the second MANOVA factors were 2 (viewing angle) $\mathrm{x}$ 3 (movement speed). All data satisfied the normality criterion as verified using the Kolmogorov-Smirnov test.

\section{E. Effects of head/torso inclination}

The analysis showed that the head/torso inclination factor had a significant effect on the ratings $(F(4,13)=23.5, p<0.001, \Lambda=0.1)$. This effect was pronounced both for arousal, $F(2,29)=$ $49.9, p<0.001$, and for valence, $F(1,24)=$ $45.2, p<0.001$. The post-hoc Bonferroni comparisons for the arousal ratings showed that the difference between head/torso down $(\mathrm{M}=2.5, \mathrm{SD}=0.3)$ condition was significantly lower $(p<0.001)$ than normal the head/torso condition $(\mathrm{M}=5.4, \mathrm{SD}=0.3)$, and the head/torso up condition $(\mathrm{M}=6, \mathrm{SD}=0.3)$. The same comparisons for the valence ratings showed significant differences for all three conditions. The head/torso up condition was perceived as most pleasant followed by the normal head/torso position, and head/torso down. The means were $\mathrm{M}=6.7, \mathrm{SD}=0.2 ; \mathrm{M}=5.9, \mathrm{SD}=$ 0.3 ; and $\mathrm{M}=2.8, \mathrm{SD}=0.5$, respectively. No effect of the viewing angle, or interaction between the angle and the head/torso position reached significance.
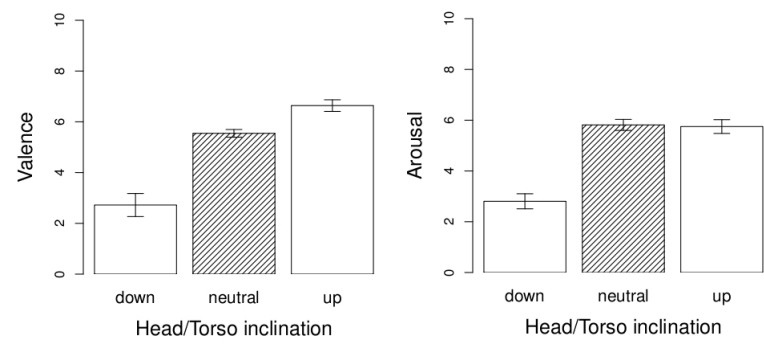

Fig. 2. Valence and arousal rating for Head/Torso inclination. Error bars indicate standard error. Valence rating 0 indicates a very sad emotional state, rating 10 a very happy state. Arousal rating 0 indicates a low arousal state, arousal rating 10 indicates a high arousal state.

\section{F. Effects of walking speed}

The movement speed factor reached significance at $F(4,13)=41.1, p<0.001, \Lambda=0.07$. This effect was caused only by the arousal ratings, $F(2,27)=$ $58.6, p<0.001$. The post-hoc Bonferroni comparisons for the arousal ratings showed that fast speed motion $(\mathrm{M}=8.1 \mathrm{SD}=0.2)$ was significantly different from the normal speed $(\mathrm{M}=5.4 \mathrm{SD}=0.3)$, and from 
the slow speed conditions $(\mathrm{M}=4.2 \mathrm{SD}=0.3)$. No effect of viewing angle, or interaction between the angle and the movement speed reached significance.
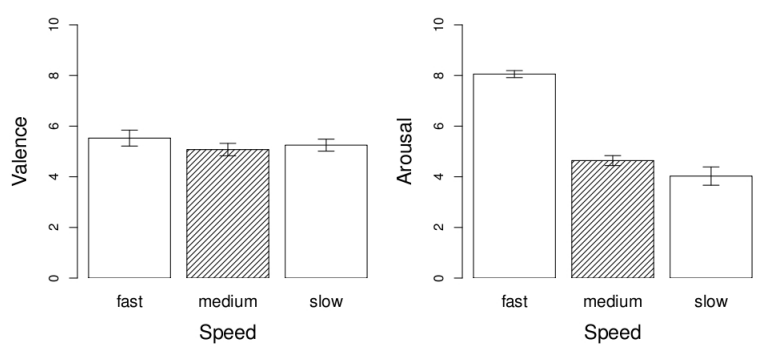

Fig. 3. Valence and arousal rating for Head/Torso inclination Error bars indicate standard error. Valence rating 0 indicates a very sad emotional state, rating 10 a very happy state. Arousal rating 0 indicates a low arousal state, arousal rating 10 indicates a high arousal state.

When locating the animations in the circumplex model of valence and arousal (Figure 4), we see that a wide area is covered, indicating the power of the head/torso inclination and speed parameters to express a range of emotional states. The coordinates that are not sufficiently covered yet, are the combinations of high valence/low arousal, and low valence/high arousal.

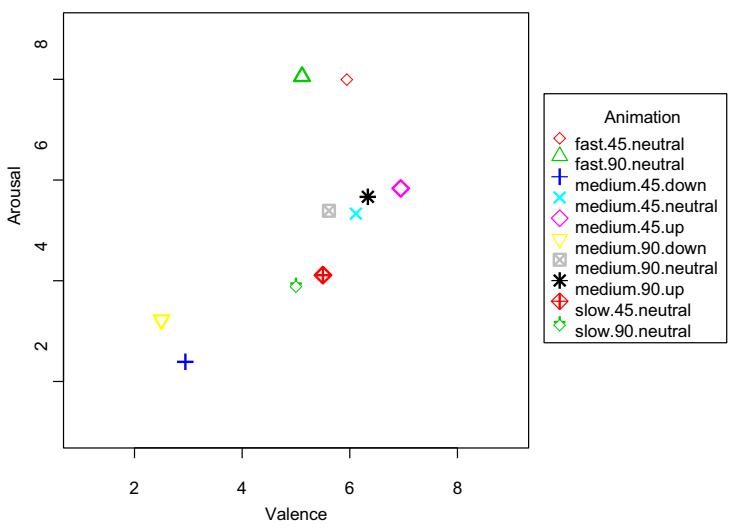

Fig. 4. Distribution of the animations in the circumplex. The legend indicates the stimuli parameter space of the different animations: $<$ speed $>$. $<$ viewing angle $>$. $<$ head/torso inclination $>$. The speed parameter is defined as Fast $=1.4 \mathrm{~m} / \mathrm{sec}$, Medium $=0.75 \mathrm{~m} / \mathrm{sec}$ and Slow $=0.5 \mathrm{~m} / \mathrm{sec}$. The viewing angle varies between profile view $=90$ degrees, and rotated frontal view $=45$ degrees. The parameter for the head/torso inclination varies between Neutral $=0$ degrees, $\mathrm{Up}=+15$ degrees and Down $=-55$ degrees.

\section{DISCUSSION}

Our results show that participants assigned distinct emotional states to animations of a walking person that only differed in the erection of the posture, and walking speed. An upright head/torso position was significantly related with a positive emotional state or high valence, a lower position with a more unpleasant emotional state. Even small changes in head/torso position of 15 degrees induced a significantly different perception of the emotional quality. This is indicative of the high sensitivity of humans in relating subtle differences in body language to internal states. Next to the valence, also the arousal rating was significantly affected by the body posture: Animations with negative head/torso inclinations were perceived as less aroused compared to body postures with more upright head/torso positions. These results are in line with studies showing that especially the static configuration of the upper body part code important features responsible for the perception of emotional states [38], [41]. While the valence rating differed in all three head/torso conditions, in the arousal rating we only observed significant difference for the most extreme negative head/torso inclination . This finding suggest that only extreme down positions of the head clearly code low values of arousal, which is in line with other studies that found that depressive states were characterized by non-erected postures [56], [38]. The different walking speeds had a clear effect on the perception of arousal: Higher speed yielded higher arousal ratings compared to slower movements. This means that the velocity of the body movements does provide information about the magnitude of an emotional state of a person. This finding is in line with recent studies showing that the velocity of body movements codes the intensity of a perceived emotional state [40], [38]. Contrary to this observation, the speed had no effect on the valence rating of the perceived emotion. If we are searching for canonical parameters that control the expression of emotions in animations, we aim at finding parameters that are independent of the angle from which their are seen. Indeed, our results show that the emotional quality of the animations generated based on the chosen set of parameters are independent of the viewing angle.

\section{CONCLUSION}

The identification and empirical evaluation of canonical parameters that control the expression of emotions in locomotive behavior is the main contribution of this study. Our results are coherent with previous work, showing that upright upper body postures are perceived as emotionally more positive and forward leaning postures more negative [32], [26], [38], and studies that found associations between "dropped head" positions and sadness [2], [57]. The perception of the arousal state can be related to a variation of the velocity of the movement, which is in line with findings from [56], [58]. The results of our study confirm previous results stating that the intensity of a perceived emotion is directly linked to the velocity of the identified body gesture [40]. Our study therefor supports the hypothesis proposed by others that the 
static configuration of the body parts, especially the upped back, shoulders and head inclination valence value [38], [41], while the kinematic dimension codes the intensity of the emotion [40].

Even though context [2], [53], [21] and facial expressions [54], [55] play an important role in giving meaning to bodily expression, our results show that people recognize distinguishable emotional states of a moving person independent of those two factors. Hence, we show that the characteristic of locomotion by itself can convey emotional states.

These findings are important as they allow us to build virtual characters whose emotional expression is recognizable at distances larger than those at which facial expression can be decoded. Additionally, the moving characters can keep their emotional state during an extended period of time. This is important since observing an isolated emotive face over a long time can be perceived as a non-natural behavior. The understanding of both of the mentioned aspects is of relevance for the construction of avatars that interact with users in virtual worlds or in environments such as CAVEs [59] and mixed-reality spaces such as the eXperience Induction Machine [60]. Future work will include the investigation of additional parameters that allow to cover the entire circumplex space. Additionally, we plan to apply our finding to the control of the emotional expression of a real-world robotic platforms such as the humanoid robot iCub [61].

\section{ACKNOWLEDGMENT}

The authors would like to thank Nina Valkanova and Julian Bonequi for their professional support in the production of the animations. This work was supported by CEEDS project (Project No. FP7 ICT258749), the European Communitys Seventh Framework Programme FP7/2007-2013.

\section{REFERENCES}

[1] W. H. Dittrich and A. P. Atkinson, Affective Computing: Focus on Emotion Expression, Synthesis and Recognition. Vienna: I-Tech Education and Publishing, 2008, ch. The Perception of Bodily Expressions of Emotion and the Implications for Computing, pp. $157-184$.

[2] H. Wallbott, "Bodily expression of emotion," European Journal of Social Psychology, vol. 28, no. 6, pp. 879-896, 1998.

[3] R. Lazarus and F. Susan, Stress, Appraisal, and Coping. New York: Springer Publishing Company Inc., 1984.

[4] A. Damasio, B. Everitt, and D. Bishop, "The Somatic Marker Hypothesis and the Possible Functions of the Prefrontal Cortex," Philosophical Transactions: Biological Sciences, pp. 1413-1420, 1996.

[5] J. LeDoux, "Emotion circuits in the brain," Annual Review of Neuroscience, vol. 23, no. 1, pp. 155-184, 2000.

[6] K. R. Scherer and P. Ekman, Approaches to Emotion. Hillsdale, NJ: Lawrence Erlbaum Associates, 1984, ch. Expression and the nature of emotion, pp. 319-344.

[7] P. Ekman, "Facial expression and emotion." American Psychologist, vol. 48, no. 4, pp. 384-392, 1993.

[8] C. Izard, "Innate and universal facial expressions: Evidence from developmental and cross-cultural research." Psychological Bulletin, vol. 115, no. 2, pp. 288-299, 1994.
[9] W. E. Rinn, "The neuropsychology of facial expression: A review of the neurological and psychological mechanisms for producing facial expressions," Psychological Bulletin, vol. 95, no. 1 , pp. $52-77,1984$.

[10] K. R. Scherer, R. Banse, and H. G. Wallbott, "Emotion Inferences from Vocal Expression Correlate Across Languages and Cultures," Journal of Cross-Cultural Psychology, vol. 32, no. 1, pp. 76-92, 2001.

[11] D. A. Sauter, F. Eisner, P. Ekman, and S. K. Scott, "Crosscultural recognition of basic emotions through nonverbal emotional vocalizations," Proceedings of the National Academy of Sciences of the United States of America, vol. 107, no. 6, pp. 2408-2412, 2010

[12] A. J. Fridlund, B. Apfelbaum, G. Blum, D. Brown, J. Balakrishnan, J. Loomis, G. Mchugo, M. Platow, and P. Rozin, "Sociality of Solitary Smiling : Potentiation by an Implicit Audience," Journal of Personality and Social Psychology, vol. 60, no. 2, pp. 229-240, 1991.

[13] R. E. Kraut and R. E. Johnston, "Social and Emotional Messages of Smiling : An Ethological Approach," Journal of Personality and Social Psychology, vol. 37, no. 9, pp. 15391553, 1979.

[14] P. Ekman and W. Friesen, Facial Action Coding System: A Technique for the Measurement of Facial Movement. Palo Alto: Consulting Psychologists Press, 1978.

[15] I. Bartenfieff, Body Movement: Coping with the Environment. New York: Routledge, 1980.

[16] J. Pforsich, Handbook for Laban Movement Analysis. New York: Janis Pforsich, 1977.

[17] R. L. Birdwhistell, Expressions of the emotion in man. New York: International University Press, 1963, ch. The kinesic level in the investigation of emotions, pp. 123-139.

[18] - Kinesics and context. Philadelphia: University of Pennsylvvania Press, 1970.

[19] S. Jolly, "Understanding body language: Birdwhistell's theory of kinesics," Corporate Communications: An International Journal, vol. 5, no. 3, pp. 133-139, 2000.

[20] A. Mehrabian, Nonverbal communication. New Jersey: Aldine Transaction Publishers, 1972

[21] M. E. Kret and B. de Gelder, "Social context influences recognition of bodily expressions." Experimental Brain Research, vol. 203, no. 1, pp. 169-80, 2010.

[22] R. Walk and C. Homan, "Emotion and dance in dynamic light displays," Bulletin of the Psychonomic Society, vol. 22, no. 5, pp. 437 - 440, 1984.

[23] A. Camurri, I. Lagerlöf, and V. Gualtiero, "Recognizing emotion from dance movement: comparison of spectator recognition and automated techniques," International Journal of Human-Computer Studies, vol. 59, no. 1-2, pp. 213-225, 2003.

[24] R. Blake and M. Shiffrar, "Perception of human motion." Annual review of psychology, vol. 58, pp. 47-73, 2007.

[25] K. Walters and R. Walk, "Perception of emotion from body posture," Bulletin of the Psychonomic Society, vol. 24, no. 5, pp. 329-329, 1986.

[26] M. Coulson, "Attributing Emotion to Static Body Postures: Recognition Accuracy, Confusions, and Viewpoint Dependence," Journal of Nonverbal Behavior, vol. 28, no. 2, pp. 117-139, 2004.

[27] A. Garcia-Rojas, F. Vexo, D. Thalmann, A. Raouzaiou, K. Karpouzis, and S. Kollias, "Emotional body expression parameters in virtual human ontology," First International Workshop on Shapes and Semantics, 2008.

[28] G. Johansson, "Visual perception of biological motion and a model for its analysis," Perception and Psychophysics, vol. 14, pp. 201-211, 1973.

[29] T. J. Clarke, M. F. Bradshaw, D. T. Field, S. E. Hampson, and D. Rose, "The perception of emotion from body movement in point-light displays of interpersonal dialogue," Perception, vol. 34, no. 10, pp. 1171-1180, 2005.

[30] F. E. Pollick, H. M. Paterson, A. Bruderlin, and A. J. Sanford, "Perceiving affect from arm movement," Cognition, vol. 82, no. 2, pp. B51-B61, 2001.

[31] W. H. Dittrich, T. Troscianko, S. E. G. Lea, and D. Morgan, "Perception of emotion from dynamic point-light displays 
represented in dance," Perception, vol. 25, no. 6, pp. 727-738, 1996.

[32] S. Schouwstra and J. Hoogstraten, "Head position and spinal position as determinants of perceived emotional state," Perceptual and Motor Skills, no. 2, pp. 673-674, 1995.

[33] A. Kleinsmith and N. Bianchi-Berthouze, "Recognizing affective dimensions from body posture," in International Conference of Affective Computing and Intelligent Interaction, Lisboa (Portugal), 2007, pp. 48-58.

[34] A. Kleinsmith, P. De Silva, Bianchi-Berth, and N. Ouze, "Cross-cultural differences in recognizing affect from body posture," Interacting with Computers, vol. 18, no. 6, pp. 13711389, Dec. 2006.

[35] P. De Silva and N. Bianchi-Berthouze, "Modeling human affective postures: an information theoretic characterization of posture features," Computer Animation and Virtual Worlds, vol. 15, no. 3-4, pp. 269-276, Jul. 2004.

[36] A. Camurri, S. Hashimoto, K. Suzuki, and R. Trocca, "KANSEI analysis of dance performance," in IEEE International Conference on Systems, Man and Cybernetics. Ieee, 1999, pp. 327-332.

[37] S. Kamisato, S. Odo, Y. Ishikawa, and K. Hoshino, "Extraction of Motion Characteristics Corresponding to Sensitivity Information Using Dance Movement," Computational Intelligence, vol. 8, no. 2, 2004.

[38] C. L. Roether, L. Omlor, A. Christensen, and M. A. Giese, "Critical features for the perception of emotion from gait," Journal of Vision, vol. 9, no. 6, pp. 1-32, 2009.

[39] S. M. Thurman, M. A. Giese, and E. D. Grossman, "Perceptual and computational analysis of critical features for biological motion," Journal of Vision, vol. 10, no. 12, pp. 1-14, 2010.

[40] A. P. Atkinson, W. H. Dittrich, A. J. Gemmell, and A. W. Young, "Emotion perception from dynamic and static body expressions in point-light and full-light displays," Perception, vol. 33, no. 6, pp. 717-746, 2004.

[41] A. Atkinson, M. Tunstall, and W. Dittrich, "Evidence for distinct contributions of form and motion information to the recognition of emotions from body gestures," Cognition, vol. 104, no. 1, pp. 59-72, 2007.

[42] M. Giese and T. Poggio, "Neural mechanisms for the recognition of biological movements," Nature Reviews Neuroscience, vol. 4, no. 3, pp. 179-192, Mar. 2003.

[43] W. McDougall, An Introduction to Social Psychology. Boston: John W. Luce \& Co., 1926.

[44] S. S. Tomkins, Affective imaginary consciousness. New York: Springer, 1962

[45] C. E. Izard, Human emotions. New York: Plenum, 1977.

[46] P. Ekman, "An argument for basic emotions," Cognition \& Emotion, vol. 6, no. 3, pp. 169-200, 1992.

[47] J. Russell, "A circumplex model of affect," Journal of Personality and Social Psychology, vol. 39, no. 6, pp. 1161-1178, 1980.

[48] R. Plutchik and H. R. Conte, Eds., Circumplex Models of Personality and Emotions, Washington, DC, 1997.

[49] Autodesk Inc., San Francisco, CA, USA, "Autodesk 3ds max," 2007.

[50] Garage Games, Eugene, Oregon, USA, “Torque game engine," 2008.

[51] Psychology Software Tools, Inc., Sharspburg, PA, USA., "Eprime 1," 2007.

[52] M. Bradley and P. Lang, "Measuring emotion: the selfassessment manikin and the semantic differential," Journal of Behavior Therapy and Experimental Psychiatry, vol. 25, no. 1, pp. 49-59, 1994.

[53] H. Aviezer, R. R. Hassin, J. Ryan, C. Grady, J. Susskind, A. Anderson, M. Moscovitch, and S. Bentin, "Angry, disgusted, or afraid? Studies on the malleability of emotion perception." Psychological science: a journal of the American Psychological Society, vol. 19, no. 7, pp. 724-32, 2008.

[54] J. Van den Stock, R. Righart, and B. de Gelder, "Body expressions influence recognition of emotions in the face and voice." Emotion, vol. 7, no. 3, pp. 487-94, 2007.
[55] H. K. M. Meeren, C. C. R. J. van Heijnsbergen, and B. de Gelder, "Rapid perceptual integration of facial expression and emotional body language." Proceedings of the National Academy of Sciences of the United States of America, vol. 102, no. 45, pp. 16518-23, 2005.

[56] H. Ellgring, Nonverbal Communication in Depression. Cambridge: Cambridge University Press, 1989.

[57] P. Bull, "The interpretation of posture through an alternative methodology to role play," The British journal of social and clinical psychology, vol. 17, no. 1, pp. 1-6, 1978.

[58] P. Ekman and W. Friesen, "Detecting deception from the body or face." Journal of Personality and Social Psychology, vol. 29, no. 3, pp. 288-298, 1974.

[59] C. Cruz-Neira, D. J. Sandin, T. A. DeFanti, R. V. Kenyon, and J. C. Hart, "The cave: Audio visual experience automatic virtual environment," Communications of the ACM, vol. 35, no. 6, pp. 64-72, 1992.

[60] U. Bernardet, M. Inderbitzin, S. Wierenga, A. Väljamäe, A. Mura, and P. F. M. J. Verschure, "Validating presence by relying on recollection: Human experience and performance in the mixed reality system XIM," The 10th International Workshop on Presence., 2008.

[61] G. Sandini, G. Metta, and D. Vernon, "The iCub Cognitive Humanoid Robot : An Open-System Research Platform for Enactive Cognition Enactive Cognition : Why Create a Cognitive Humanoid," in 50 Years of Artificial Intelligence, ser. Lecture Notes in Computer Science, M. Lungarella, F. Iida, J. Bongard, and R. Pfeifer, Eds. Springer Berlin / Heidelberg, 2007, vol. 4850, pp. 358-369. 